\title{
El papel de la universidad en la conservación y promoción de de la cultura en el adulto mayor
}

The role of the university in the preservation and promotion of culture in the elderly

DOI: $10.46932 / \mathrm{sfjdv2n5-107}$

Received in: Oct 1st, 2021

Accepted in: Dec 30th, 2021

\author{
Ricardo Pereira Nodarse \\ Master en Ciencias de la Educación. \\ Profesor Auxiliar. \\ Universidad Marta Abreu de Villa Clara. \\ Carretera Central Nro 101 Cascacal Villa Clara. \\ Correo electrónico: ricardopn@uclv.cu \\ Jorge Jesùs Dìaz Gàlvez \\ Master en Educación Superior. \\ Profesor Auxiliar. \\ Universidad Marta Abreu de Villa Clara. \\ Camilo Cienfuegos Nro 158 Manacas Villa Clara \\ Correo electrónico:jjgd@uclv.edu.cu \\ Maidelìn Martìnez Rivero \\ Master en Educación Superior. \\ Profesor Asistente. \\ Universidad Marta Abreu de Villa Clara. \\ Maceo Norte Nro 19 Manacas Villa Clara \\ Correo electrónico: mayi@uclv.edu.cu \\ Ana Margarita Castro Leal \\ Licenciada en Contabilidad y Finanza \\ Profesor Asistente. \\ Universidad Marta Abreu de Villa Clara \\ Frank Pais Nro 34 Manacas Villa Clara \\ Correo electrónico: amcl@uclv.edu.cu

\section{Angela Olina Brito Sotolongo} \\ Master en Educación Superior. \\ Profesor Asistente. \\ Universidad Marta Abreu de Villa Clara. \\ Frank Pais Nro 35 Manacas Villa Clara \\ Correo electrónico: angelab@uclv.edu.cu

\section{Arlety Martìnez Benavidez} \\ Ingeniera Geóloga \\ Profesor Asistente \\ Universidad Marta Abreu de Villa Clara \\ Calle Segunda Nro 54 Santo Domingo Villa Clara
}


Correo electrónico: arle@uclv.edu.cu

\author{
Mariluz Martínez Espinosa \\ Licenciada en Matemática \\ Categoría Docente: Profesor Asistente. \\ Universidad Marta Abreu de Villa Clara \\ Centro Comunal Sabino Hernández Nro S/N Manacas Villa Clara \\ Correo electrónico: marilys @uclv.edu.cu \\ Yuleidys Pérez Ruiz \\ Licenciada en Psicología \\ Profesor Asistente \\ Universidad Marta Abreu de Villa Clara \\ Maceo Nro 2A2 Manacas Villa Clara \\ Correo electrónico: yuleidisp@uclv.edu.cu
}

\begin{abstract}
RESUMEN
El trabajo aborda un sistema de actividades en coordinación con los instructores de arte de cultura, joven club de computación y profesores del INDER, que responde a la necesidad de elevar la calidad de vida del adulto mayor, según las exigencias del modelo cubano. Su objetivo principal es proponer un sistema de acciones socioculturales, para elevar la calidad de vida del adulto mayor, a través actividades recreativo-culturales en el "Consejo Popular Cascajal".En su elaboración se recorren varias etapas del proceso de investigación: diagnóstico, luego se continúa con la identificación de las vocaciones por parte de los adultos, teniendo presente sus motivaciones y habilidades para el desarrollo de las actividades. Se establecen las bases para la adopción de actitudes que eleven la calidad de vida. Influyó de forma positiva en los resultados finales de de la investigación, los adultos de la tercera edad se incorporaron a las actividades culturales, artesanales, físicas y recreativas con entusiasmo y dedicación, logrando contribuir a un estado de bienestar y satisfacción plena, compensando los problemas de salud que son inherentes de esta edad y de esta forma contribuir a su calidad de vida.
\end{abstract}

Palabras claves: modelo cubano, calidad de vida, estado de bienestar, salud, acciones socioculturales.

\begin{abstract}
The work deals with a system of activities in coordination with the instructors of art of culture, young computer club and teachers of INDER, which responds to the need to raise the quality of life of the elderly, according to the demands of the Cuban model. Its main objective is to propose a system of socio-cultural actions to improve the quality of life of the elderly, through recreational-cultural activities in the "Consejo Popular Cascajal" (Cascajal Popular Council), through several stages of the research process: diagnosis, then it continues with the identification of the adults' vocations, taking into account their motivations and abilities for the development of the activities. The bases are established for the adoption of attitudes that raise the quality of life. The final results of the research were positively influenced by the fact that the elderly adults joined the cultural, handicraft, physical and recreational activities with enthusiasm and dedication, contributing to a state of well-being and full satisfaction, compensating for the health problems inherent to this age group and thus contributing to their quality of life.
\end{abstract}

Keywords: Cuban model, quality of life, state of well-being, health, sociocultural actions. 


\section{INTRODUCCIÓN}

En el siglo XX, en América Latina y el Caribe se presentaron importantes cambios demográficos con un rápido crecimiento de los grupos de personas adultas mayores con 60 años de edad y más, los cuales se han dado en condiciones socioeconómicas diferentes a las que prevalecían en los países desarrollados cuando se produjeron estos cambios.

Desde la década de 1970, las Naciones Unidas viene llamando la atención sobre el envejecimiento de la población mundial y promoviendo acciones entre sus agencias y las instituciones gubernamentales y no gubernamentales de los países que representan. La Organización Panamericana de la Salud (OPS), oficina de la Organización Mundial de la Salud (OMS) en las Américas, inició en 1997, con los esfuerzos y recursos conjuntos de un grupo de científicos y expertos de diferentes instituciones de la Región y de los siete países participantes (Argentina, Barbados, Brasil, Chile, Cuba, México y Uruguay), un proyecto multicéntrico de investigación llamado "Salud, Bienestar y Envejecimiento en América Latina y el Caribe”, (SABE), que fue el primero con estas características y permitió tener un diagnóstico de las realidades socio-demográficas y de salud de los adultos mayores en la Región.

En el caso cubano, el proceso de envejecimiento es consecuencia inevitable de la transición demográfica y la combinación de bajas tasas de fecundidad y de mortalidad, en el contexto del desarrollo social alcanzado por Cuba. Esto ha llevado a un rápido envejecimiento poblacional con un número sin precedentes de adultos mayores, y por lo tanto, de todos sus requerimientos y necesidades.

Las características de la población cubana actual, la sitúan entre los países de Latinoamérica y del mundo más envejecido. Cuba está entre los 30 países con más alta esperanza de vida al nacer (77 años). Enrique Vega, asesor regional de Envejecimiento y Salud de la Organización Panamericana de la Salud (OPS), destacó la voluntad política del Estado cubano, cuya premisa fundamental es el desarrollo del ser humano, es el único país con esa meta lograda, pues atiende en diversos programas a todos los adultos mayores, quienes suman 1820000 personas y constituyen el 15,9\% de su población, informó durante el Seminario Internacional de Atención al Adulto Mayor celebrado en el Palacio de las Convenciones en mayo del 2007.

Diversos estudios realizados arrojan que en la última etapa del ciclo de vida llamada tercera edad o adulto mayor es la etapa donde ocurre una disminución de la masa muscular, se observa un proceso de envejecimiento en las articulaciones donde cada vez los movimientos se vuelven más limitados, la inactividad física es el factor determinante en el deterioro del envejecimiento, por ende se van perdiendo las capacidades físicas ya sean condicionales ,coordinativas, y la movilidad articular o flexibilidad. Las pérdidas más apreciables estimadas en por cientos son: 
- $67 \%$ Para la agilidad.

- $58 \%$ Para las fuerzas de los miembros inferiores.

- $28 \%$ Para las fuerza de los miembros superiores.

- La pérdida de fuerza muscular es de aproximadamente de $30 \%$ y del área muscular de $40 \%$ entre la segunda y séptima década de vida.

Debido a lo antes expuesto resulta de gran interés elevar el trabajo por las instituciones de la comunidad con el propósito de atender a las personas de la tercera edad, propiciando que se sientan útiles en el hogar y puedan ayudar a la familia y a la sociedad y de esta forma elevar su calidad de vida.

A través de encuestas aplicadas a personas se pudo constatar que existe: insuficientes actividades físico- recreativas para los Adultos Mayores en el Consejo Popular Cascajal, lo cual no responde a la satisfacción de sus necesidades, motivaciones e intereses, al mismo tiempo el poblado no cuenta con un hogar de ancianos donde puedan pasar su tiempo libre

Los elementos antes descritos permiten declarar como:

Problema científico: ¿Cómo contribuir a elevar la calidad de vida del adulto mayor en el Consejo Popular Cascajal. ?

Objetivo general: Proponer un sistema de acciones socioculturales, para elevar la calidad de vida del adulto mayor, a través actividades recreativo-culturales en el "Consejo Popular Cascajal".

\section{DESARROLLO}

En Cuba se le da gran importancia al estudio de aquellos aspectos que guardan estrecha relación con el envejecimiento humano, por lo que se sustentan en la gerontología, ciencia que a su vez incluye otras como las biológicas, médicas, psicológicas y sociológicas, además se tiene presente los conocimientos científicos de avanzada, en beneficio del aumento de la expectativa de vida de los adultos mayores. El envejecimiento es un fenómeno actual que se está dando en todos los países con distintos grados de intensidad, diferentes autores de múltiples disciplinas se han interesando por el estudio de los diferentes procesos que intervienen en el envejecimiento y las posibilidades para prolongar la vida de acuerdo al nivel de desarrollo de cada uno entre ellos se destaca Foster W. (1995: 22) y Fujita F. 1995: 23), los que estudian el problema de la involución por la edad, han observado los cambios relacionados con el envejecimiento que se produce en los órganos y tejidos más disímiles del organismo humano, sin embargo, el envejecimiento no es solamente la pérdida de las funciones, ya que, al mismo tiempo en el organismo se desarrollan mecanismos compensatorios que se oponen a este fenómeno.

Foster, (1995) (22, p.18) “El envejecimiento del organismo humano además de constituir una etapa normal e irreversible de la vida, es una forma muy especial del movimiento de la materia. Es un complejo y variado proceso que depende no solamente de causas biológicas, sino también, de condiciones 
socioeconómicas en las cuales se desarrolla el individuo como ser social".

El envejecimiento a su vez produce una serie de cambios en el organismo a nivel cardiovascular, respiratorio, metabólico, músculo esquelético, motriz, que reducen la capacidad de esfuerzo y resistencia al estrés físico de las personas mayores, reduciéndose así mismo su autonomía y calidad de vida y su habilidad y capacidad de aprendizaje.

\section{LA RECREACIÓN EN EL ADULTO MAYOR}

La recreación en el adulto mayor, ha de verse más bien como una acción que proyectada desde lo social puede crear las condiciones que faciliten la elevación de la calidad de vida de estas personas, y contribuya al desarrollo de estilos de vida más saludables y autónomos a esta edad. Tiene un carácter desarrollador, por los niveles de participación y de actualización que pueden generar, emplea herramientas de acción social que permiten elevar el bienestar y la calidad de vida de las personas de la tercera edad. Las personas que envejecen adecuadamente utilizan y disfrutan de todas las posibilidades que existen en su entorno social y en sus propias personas, y están preparados para aceptar, sin claudicar, las propias limitaciones propias de su edad como son la disminución de sus fuerzas y la necesidad de recibir algún tipo de ayuda; pero no renuncian a mantener, dentro de rangos razonables y posibles, determinados niveles de independencia y autodeterminación.

En la tercera edad, la actividad física-intelectual y el interés por el entorno conducidas a través de actividades de recreación y ocio productivo, favorecen el bienestar y la calidad de vida de las personas.

Numerosas investigaciones han mostrado que la tercera edad no tiene necesariamente que ser un período de la vida en el que predomine o se haga inevitable un deterioro fatal de las capacidades físicas e intelectuales, ya que si los sujetos muestran la necesaria motivación y la intención de mantener un estilo de vida activo y productivo, y se les propician las condiciones para desenvolverse en un entorno rico y estimulante, en el cual se favorezcan experiencias de aprendizajes y se reconozcan y estimulen los esfuerzos por alcanzar determinados logros, en cuanto a participación en actividades de diversa índole, el envejecimiento puede evitarse o demorarse.

La recreación reporta múltiples beneficios para las personas de la tercera edad, entre ellas podemos citar las siguientes:

- $\quad$ Potencia la creatividad y la capacidad estética y artística.

- $\quad$ Favorece el mantenimiento de un funcionamiento psicomotriz adecuado.

- $\quad$ Fomenta la integración social.

- Mantiene, en cierto nivel, las capacidades productivas.

- Hace frente a las disminuciones y limitaciones físicas. 
- Mantiene el equilibrio, la flexibilidad y expresividad corporal.

- $\quad$ Sirve como medio de distensión y enfrentamiento activo al estrés y las tensiones propias de esta etapa de la vida.

- $\quad$ Contribuye al mantenimiento del sentimiento de utilidad y autoestima personal.

- $\quad$ Fomenta la creatividad y productividad en el uso del tiempo libre.

- Mantiene el desarrollo de la capacidad del disfrute lúdico y la apertura hacia nuevos intereses.

- Fomenta la comunicación, la amistad y el establecimiento de relaciones interpersonales ricas y variadas.

- $\quad$ Propicia el bienestar y la satisfacción personal.

\section{CARACTERIZACIÓN SOCIOCULTURAL DEL "CONSEJO POPULAR CASCAJAL"}

El Consejo Popular Cascajal se encuentra ubicado en el extremo occidental de la provincia Villa Clara en el Municipio de Santo Domingo, limita al norte con el Consejo Popular Punta Felipe, al sur con el Municipio de Rodas Provincia de Cienfuegos, por el este con el Consejo Popular de Mordazo y por el oeste con el Municipio Los Arabos de la provincia de Matanzas. Tiene una extensión de 134 Km², lo atraviesan 2 vías de comunicación importantes el ferrocarril y la carretera central, su relieve es fundamentalmente llano con suaves ondulaciones y algunas fallas, el suelo que predomina es el arenoso con una vegetación típica de sabana como son la palma cana, el espartillo y otros pastos naturales, por lo que su actividad económica es fundamentalmente agropecuaria. La población actual es de 7251 habitantes y una densidad de población de 54 habitantes por Km2. del total de la población 6622 es urbana y 629 rural, 2965 mujeres, 2965 hombres.

En educación cuenta con una Escuela primaria y un Centro Mixto (secundaria, Preuniversitario y Educación Técnica Profesional.) donde existen instructores de arte en las especialidades de plástica, danza, teatro y música. En cuanto a la cultura popular tradicional prevalece el gusto por la música campesina y mexicana, la pintura, la artesanía, contando con un potencial artístico de poetas, artesanos y pintores. Respecto a la danza prefieren los más jóvenes los bailes modernos, en las personas de mayor edad hay marcada predilección por los bailables de la época de su juventud como los boleros, sones o danzones. Existe preferencia mayormente en jóvenes y adultos por los juegos de dominó, béisbol y fútbol. En el ámbito culinario la generalidad de los pobladores prefiere la comida criolla, donde el arroz moro, la carne de cerdo, la yuca con mojo y las ensaladas de verduras conforman el menú. Las tradiciones religiosas también ocupan un lugar importante, prevaleciendo aquellas de origen católico y las concernientes al legado yoruba. Cascajal es el promotor de las parrandas en el municipio de Santo Domingo, porque fue 
el primer poblado donde se efectuaron ese tipo de fiestas, las mismas son acogidas por el pueblo con mucha alegría e entusiasmo las personas de mayor edad la disfrutaban manteniendo las tradiciones culturales de sus antepasados, las personas brindaban su apoyo en todos los aspectos. Ya que tienen como principal características la competencia entre los dos barrios El Camarón y El Teje, esta se efectuaban los 24 de febrero, siguiendo la tradición de celebrar el día del cascajalence ausente. Cada barrio tiene su color y su símbolo, El Camarón simbolizado por un Camarón, su color es el verde, con bandera de igual color. El Teje, simbolizado por un Caimán, su color es el rojo y su bandera es blanca. Estas parrandas no se han celebrado en los últimos diez años, por lo que ha propiciado que se vayan perdiendo las tradiciones culturales de la comunidad.

Las instituciones culturales son limitadas, cuenta con una casa de cultura y una biblioteca pública, las mismas no son suficientemente explotadas por la población y la participación del adulto mayor en ellas es muy restringida. Esta situación supone un punto de obligada mirada por cuanto se convierte en causa de muchas de las problemáticas que comúnmente aquejan a las personas de la tercera edad. No contar con el aliciente del disfrute sano, tan necesario en una edad de desintegración y cambio de varias funciones biológicas y psicológicas puede conllevar a la aparición de estados de frustración agudamente vivenciados, acompañados de manifestaciones depresivas y trastornos nerviosos.

\section{RESULTADOS DE LA APLICACIÓN DE LOS MÉTODOS EMPÍRICOS}

La investigación se inició en el Consejo Popular de Cascajal de una población de 1362 personas de la tercera edad. Se selecciona una muestra con carácter no probabilistico intencional de 75 adultos mayores de ellos 45 son mujeres y 30 son hombres. Se caracterizan por poseer un rango de edad entre 60 y 75 años aproximadamente, muestran interés por pertenecer al proyecto y poseen habilidades en el desarrollo de actividades de artesanía, en el baile, dramatizaciones, música y componen poesías.

A modo de lograr un conocimiento objetivo acerca de las causas asociadas a la problemática analizada y determinar las principales necesidades y potencialidades que sobre la misma presenta la muestra, se aplicó un diagnóstico a través de los siguientes métodos empíricos: observación, encuesta y entrevista.

A través de la aplicación de estos métodos se pudo definir que los principales factores asociados a la escasa participación del adulto mayor a las actividades recreativo-culturales en la comunidad están dados por una incongruencia entre el contenido de las propuestas ofertadas en relación con las peculiaridades de su edad. De este modo, las principales necesidades, intereses y expectativas de este grupo etario en relación a la recreación y el disfrute en forma general no quedan reflejadas en estas actividades, donde el contenido y la idea de recreación están más en función de los jóvenes. En relación 
a ello, se pudieron detectar necesidades y potencialidades, que devienen punto de partida para el diseño del sistema de acciones orientadas a solventar la problemática, las cuales se relacionan a continuación:

\section{Necesidades:}

- Carencia de un espacio en la comunidad dirigido propiamente a ofertar actividades recreativoculturales al adulto mayor.

- Insuficiente inclusión de propuestas acordes a la edad dentro del grupo de actividades recreativoculturales que se realizan en la comunidad.

- Pérdida de las tradiciones culturales.

- Potencialidades:

- Reconocimiento de la necesidad de espacios recreativos por algunas de las instituciones de la comunidad.

- Motivación de las personas de la tercera edad por participar en actividades recreativas.

- Presencia en la comunidad de lugares aprovechables para la realización de actividades recreativoculturales.

\section{APLICACIÓN DEL SISTEMA DE ACCIONES Y ANÁLISIS DE LOS RESULTADOS}

Para la aplicación del sistema de acciones se procede a realizar las coordinaciones pertinentes para su puesta en práctica, ofreciéndoles orientaciones a los responsables de las tareas, para llevarlas a cabo, determinado por los elementos recogidos en los diversos instrumentos aplicados y descritos en el diagnóstico de necesidades.

\section{Actividades Específicas}

\section{Tarea 1. Artesanía}

Nombre: Manos de Ángeles

Objetivo: Proponer un conjunto de actividades artesanales que propicien el desarrollo de la calidad de vida del adulto mayor al sentirse útiles.

Responsable: Norma Hernández Rodríguez.

El desarrollo de la artesanía en la localidad data de tiempos muy remotos, dando lugar al surgimiento de los artistas populares, ellos son los artesanos que han aprendido la técnica y el empleo de los materiales en el seno familiar convirtiéndose en portadores de una tradición que han transmitido de padres a hijos, de generación a generación. 


\section{Artículos elaborados:}

Sombreros, carteras, abanicos, doiles, pamelas, objetos confeccionados empleando semillas y madera (barcos, flores, juguetes, etc.). Objetos confeccionados empleando tela: Jabas, agarraderas, cojines, muñecas, entre otros.

Materiales empleados: Guano de yarey, hilo, semillas, recortes de madera, cola de pegar, conchas, caracoles, Arcilla, papel maché, goma de pegar, tempera, olió.

\section{Tarea 2. Cultura:}

\section{Nombre: Eterna juventud}

Objetivo: Proponer un sistema de acciones culturales que logren incrementar la calidad de vida del adulto mayor perteneciente al consejo popular Cascajal.

Responsable: Carlos L. Marrero Miranda

La recreación es aquella actividad humana, libre, placentera, efectuada individual o colectivamente, destinada a perfeccionar al hombre. Le brinda la oportunidad de emplear su tiempo libre en ocupaciones espontáneas u organizadas, que le permiten volver al mundo creador de la cultura, que sirven a su integración comunitaria.

La cultura es uno de los eventos culturales más importantes que acontecen en la localidad donde se hacen homenajes a personalidades de la cultura que ya no se encuentran entre nosotros y se premia la labor de los artistas más destacados de la comunidad. El cascajalense ausente, fiesta que se celebra el 24 de febrero el día más importante para todos los habitantes, ya que se conmemora un aniversario más de la fundación de la iglesia católica en el año 1937. Con motivo de esta celebración se realizan festejos religiosos para agasajar al Santo patrón del pueblo "Jesús de Nazareno", fiesta que se sale del marco religioso y terminaron con una celebración popular. Ese día se les hace una invitación especial a todos los cascajalenses que ya no viven en el poblado y se hacen intercambios de experiencias entre ellos y se recuerda a aquellos que ya no están y participan de las parrandas. En los últimos años no se están realizando las parrandas por lo que es un reclamo de los adultos mayores rescatar estas tradiciones culturales de la comunidad.

Ejemplo de algunas de las actividades artísticas que realizan los abuelos.

- Obras de teatro: Aceituna (adaptación), Filosofía africana, Novio.

- Dramatización: La edad, Tengo, La muralla.

- Declamación: Poesías, monólogos, décimas, poema, cuartetas.

- Composición: Poesías, monólogos, décimas, poema, cuartetas.

- Evento Panorama Teatral.

- Festival Tradicional Campesino. 
- Compartiendo entre amigos.

- Jornada de la Cultura.

- Exposición Artesanal.

- Evento Cultura Comunitaria en el Barrio.

- Actividad Danzoneando en el Barrio.

- Giras por Centros de trabajo de la Comunidad.

- Talleres de Apreciación Literaria.

- Evento de Tradiciones Campesinas,

\section{Tarea 3. GeroClub}

\section{Título: Sin límites a la esperanza...}

Objetivo: Desarrollar un proceso de formación a sus miembros orientada a la creación y ampliación de la cultura informática y gerontológico a favor del desarrollo personal y comunitario.

Responsable: Bárbara María Cepero Gálvez

Desde hace más de una década en los Joven Club de Electrónica y Computación se realizan trabajos con personas de este grupo etario. En el evento INFOCOMUNIDAD emerge la idea por parte del Equipo Nacional de hacer de este suceso un movimiento nacional.

El Geroclub de la comunidad Cascajal cuenta con 63 miembros, los que se han ido incorporando a otras actividades de la comunidad con estos mismos fines y ha devenido en un programa comunitario con el adulto mayor.

\section{Principales actividades.}

- Formación de los miembros en Informática incluyendo contenidos relacionados con las necesidades del adulto mayor.

- Utilización de las herramientas informáticas a favor de soluciones comunitarias.

- Actividades intergeneracionales que favorezcan la cultura informática, gerontológico y general integral.

- Talleres temáticos presénciales y virtuales.

- Intercambio y diseminación de información que favorezca el rescate de la historia y las tradiciones locales.

- Fomento de actividades que contribuyen al funcionamiento e integración con otras organizaciones de los adultos mayores 


\section{Tarea 4. Salud}

\section{Nombre: Todos juntos por la salud}

Objetivo: Fomentar acciones de promoción y prevención de salud en el adulto mayor para disminuir la morbi-mortalidad por enfermedades transmisibles y crónicas no transmisibles.

\section{Responsable: Dra Marisela Peón Rodríguez}

\section{Participantes: 69}

Consiste en la elaboración de materiales educativos (Volantes, Plegables, Guías prácticas y Juegos Didácticos), confeccionados con recursos locales; los que se han distribuido entre los adultos. Algunos juegos didácticos se encuentran en soporte digital en el joven club donde pueden interactuar con ellos.

Ejemplos de algunas de las actividades que se realizan con las personas de la tercera edad.

- Conferencia sobre la prevención de enfermedades transmisibles.

- Taller: "La sexualidad en la tercera edad".

- Taller: "Técnicas antiestrés y tercera edad".

- Taller: "Estilo de vida del Adulto Mayor

- Preparación a la familia en la atención de las personas en la tercera edad.

- Medidas Profilácticas para la eliminación de los vectores, priorizando al mosquito Aedes aegypti y albopictus, transmisores de enfermedades como: fiebre amarilla, dengue, zika y chikungunya.

\section{Tarea 5. Actividad Física comunitaria}

\section{Nombre: Hermosas margaritas}

Objetivo: Proponer un conjunto de actividades físicas y recreativas que logren acrecentar la calidad de vida del adulto mayor del Consejo Popular Cascajal.

\section{Responsable: Lic: Migdaly Hernández Pérez}

La actividad física bien dirigida es una opción saludable, económica y asequible que permite aumentar la calidad de vida del adulto mayor desde el punto de vista funcional, psicológico y social, la elaboración de un programa integral debe considerar el diagnóstico y su evaluación.

El envejecimiento es un proceso complejo y variado que depende no solo de las causas biológicas sino también de las condiciones sociales de vida, por eso es característico de las funciones de los profesores que atienden la actividad física comunitaria lograr la conservación de la salud, la capacidad de trabajo, propiciando una longevidad placentera en el adulto mayor. El programa de atención comunitaria de adulto mayor dentro de sus objetivos específicos está promover cambios de estilos de vida, hábitos y costumbres que favorecen la salud y así retardar la aparición de enfermedades que puedan conllevar a su evolución. 
Ejemplos de actividades:

- Excursiones a centros recreativos.

- Caminatas.

- Campeonato de dominó.

- Celebración de cumpleaños colectivos.

- Festival recreativo del adulto mayor.

- Realización de ejercicios físicos en el gimnasio biosaludable.

- Campeonato de dama.

- Campeonato de de ajedrez.

- Competencia de agilidad mental.

- Conferencia sobre: El empleo de la recreación y el uso del tiempo libre en el hogar.

- Tabla gimnástica

- Celebración del día de la mujer, día de los padres, fin de curso, fin de año.

\section{Tarea 6. CUAM Cascajal}

\section{Nombre: Los desafíos de la educación del adulto mayor}

\section{Participantes: 24}

Objetivo: Contribuir a lograr una mejor calidad de vida en el adulto mayor en el Consejo Popular de Cascajal.

Los temas tratados en la Universidad del Adulto Mayor" abordan generalidades relevantes referidas al envejecimiento activo; cuestiones relativas al funcionamiento del cuerpo y la mente, la personalidad del adulto mayor, las relaciones interpersonales y familiares, la sexualidad y la calidad de vida en la etapa. Las recomendaciones que aquí se ofrecen contribuirán al autoconocimiento, para optimizar las capacidades físicas y psicológicas, la compensación y activación de las funciones vitales, el mejoramiento de las relaciones familiares y sociales, el manejo de conflictos y solución de problemas; para el logro de una adecuada relación del adulto mayor consigo mismo, con los demás y con los contextos de actuación.

En el desarrollo de los temas se ha empleado una metodología teórico - práctica, donde se explican las cuestiones generales del contenido en un lenguaje asequible para el lector, pero respetando en todo momento el rigor conceptual que el tratamiento del tema requiere; además se presentan recomendaciones y sugerencias para el desarrollo de habilidades y modos de actuación que favorezcan el desempeño del adulto mayor; como complemento se plantean ejercicios evaluativos donde se privilegia la auto evaluación y la coevaluación (compartida con los otros), lo que permitirá a los alumnos valorar su propio desempeño, 
sus potencialidades y logros, de manera que autorregulen su aprendizaje y se conviertan en potenciadores de su propio desarrollo. Entre los temas tratados están:

Teama \# 1: Desarrollo Humano

Teama \#2 Salud para Todos

Teama \#3. Cultura contemporánea en el nuevo milenio

Teama \#4 Seguridad y Asistencia Social

Teama \#5 Educación y Desarrollo

Teama \#6 Utilización del Tiempo Libre

Teama \#7 Computación

Teama \#8 La estética y la belleza en la Tercera Edad.

En el desarrollo del proceso de preparación del trabajo y de la experiencia constada en la actividad en las Cátedra de la Universidad del Adulto Mayor (CUAM), se puede concluir diciendo que:

La Educación en Valores Humanos es un proceso que tiene grandes posibilidades de materializarse hacía la comunidad con el trabajo del adulto mayor vinculado a los grupos de trabajo comunitario integrado. El trabajo comunitario integrado encuentra un portador material en los adultos mayores en cada área de residencia, contribuye a elevar la autoestima y la calidad de vida y el sentirse útil en las personas de la tercera edad al demostrarse a sí mismo que puede y debe contribuir a pesar de sus años con el desarrollo espiritual de la sociedad.

\section{Otras acciones de carácter general.}

- Fundación de una tertulia literaria y de narración oral.

- Excursiones y visitas a lugares históricos y recreativos.

- Club "Amigos del arte".

- Peña "El deporte en el adulto mayor".

- Fórum por un medio ambiente sano en la comunidad.

- Celebración de cumpleaños colectivos.

- Colaboración con el adulto mayor que presenta más necesidades.

- Realizar actividades culturales recreativas para todos los grupos etáreos teniendo presente sus preferencias.

- Formación y funcionamiento de grupos artísticos.

- Actuación de los grupos culturales en las actividades de la comunidad.

- Realizar talleres de artesanía.

- Diseñar y confeccionar boletines de salud.

- Celebrar anualmente la fiesta del cascajalense ausente el 24 de febrero. 
- Creación y funcionamiento de círculos de interés con los estudiantes de primaria y del Centro Mixto.

Con la aplicación del sistema de acciones se alcanzan los objetivos propuestos en la investigación visto reflejada en el desarrollo de la calidad de vida de los adultos en el Consejo Popular Cascajal, como se muestra en la siguiente tabla.

\section{COMPARACIÓN DE LOS RESULTADOS DE ETAPA INICIAL Y FINAL}

\begin{tabular}{|l|l|l|l|l|l|l|l|}
\hline Indicadores & Participantes & \multicolumn{2}{|l|}{ Etapa Inicial de la investigación } & \multicolumn{2}{l|}{ Etapa Final de la investigación } \\
\hline & & Alto & Medio & Bajo & Alto & Medio & Bajo \\
\hline $\begin{array}{l}\text { Funcionamiento } \\
\text { psicomotriz adecuado }\end{array}$ & 75 & 5 & 10 & 60 & 35 & 40 \\
\hline Integración social & 75 & & & & \\
\hline $\begin{array}{l}\text { Bienestar y satisfacción } \\
\text { personal }\end{array}$ & 75 & 15 & 20 & 15 & 50 & 60 & 15 \\
\hline $\begin{array}{l}\text { Sentimiento de utilidad y } \\
\text { autoestima }\end{array}$ & 75 & 18 & 20 & 37 & 60 & 15 \\
\hline $\begin{array}{l}\text { Mantiene el equilibrio, la } \\
\text { flexibilidad y expresividad } \\
\text { corporal }\end{array}$ & 75 & 10 & 15 & 50 & 65 & 0 \\
\hline $\begin{array}{l}\text { Productividad en el uso del } \\
\text { tiempo libre }\end{array}$ & 75 & 10 & 15 & 50 & 65 & 0 \\
\hline
\end{tabular}

La instrumentación del sistema de acciones, evidencia que ha provocado un cambio significativo en el desarrollo de la calidad de vida del adulto mayor en la comunidad de Cascajal visto reflejado en: Los adultos mayores se sienten felices y lo expresan al poder hacer las cosas que pensaban y que por la edad no les estaba permitido. Mejoran los niveles de estrés y se sienten útiles, sienten que con su desempeño y sus patologías disminuyen, aumenta la autoestima y pasan a ser miembros activos de su propio grupo, el deporte, la lúdica y la recreación permiten mejorar el funcionamiento motriz, el adulto mayor se siente con altos niveles de autoconfianza, la actividad ayuda a los ancianos a poner a funcionar y a trabajar su cuerpo, su mente a través de tareas que ayudan a mejorar la calidad de vida y se crean espacios de sana convivencia, salen de la rutina y ven espacios de vida diferentes, contribuye con la tranquilidad y la paz interior, crea espacios donde ellos liberan tensiones por medio de la actividad, el adulto al sentirse útil, empieza a recordar amigos y saberes y empiezan a participar en diferentes eventos porque sienten que pueden aportar, empiezan a hacer ejercicios, participan en danzas, competencias, juego y están activos, disminuye la soledad y las dolencias, logran un mejoramiento en su desarrollo físico y funcionamiento psicológico, como parte de la expresión corporal y artística, se sienten aceptados y la autoestima se eleva, Aprenden a tener aprecio a su edad y mejora su calidad de vida, se fortalece la autoestima, a la vez que se favorece las relaciones familiares, les aumenta la fuerza vital y les ayuda a conservar la salud, los estimula y los hace sentir que son importantes para la sociedad y que se les tiene 
en cuenta, permite que desarrollen las capacidades y habilidades que ya se estaban ocultando, se observan serenos, duermen más, sus movimientos se hacen menos lentos y se sienten jóvenes nuevamente.

\section{CONCLUSIONES}

- La determinación de los fundamentos teórico - metodológicos permitió conocer que la vinculación del adulto mayor a actividades físico-recreativas en el ámbito comunitario es una problemática de interés a nivel internacional, considerando su impacto en un envejecimiento saludable.

- El diseño de un sistema de acciones para elevar la calidad de vida del adulto mayor en el Consejo Popular Cascajal puede constituir una herramienta útil para su vinculación a las actividades recreativas, artesanales, físicas y culturales de la comunidad.

- El sistema de acciones influyó de forma positiva en los resultados finales de de la investigación, los adultos se incorporaron a las actividades culturales, artesanales, físicas y recreativas con entusiasmo y dedicación, logrando contribuir a un estado de bienestar y satisfacción plena, compensando los problemas de salud que son inherentes de esta edad y de esta forma contribuir a su calidad de vida. 


\section{BIBLIOGRAFÍA}

Anuario Demográfico de Cuba 2000. Centro de Estudios de Población y Desarrollo. Oficina Nacional de Estadísticas. La Habana. 2001.

Cuba: Proyección de la Población. Nivel Nacional y Provincial. Período 2006-2030. Centro de Estudios de Población y Desarrollo. Oficina Nacional de Estadísticas. La Habana. 2005.

Colectivo de autores, (1999), Orientaciones Metodológicas para el Adulto Mayor. INDER, Ciudad de la Habana, Cuba.

Devesa Colina, E. (1996). Geriatría y Gerontología. Ciudad de La Habana. Editorial Científico-Técnica. Foster,W.(1995) Endocrinology.Ed. 2da, USA.1500.

Fujita,F.(1995) Phisyotherapeut treatment. Ed. 1ra.USA, 350.

Hernández Sampieri, R., Fernández-Collado, C., \& Baptista Lucio, P. (2006). Metodología de la Investigación. Istapalapa: McGraw-Hill.

Iglesias Durán, O. (2001). Lo psicológico en el envejecimiento. En F. Núñez Villavicencio, Psicología y salud (págs. 145-156). Ciudad de la Habana: Ciencias Médicas.

Martínez Casanova, M. (2011). Promoción Sociocultural II. La Habana: Editorial Félix Varela.

Orosa Fraíz, T. (2003). La tercera edad y la familia. La Habana: Félix Varela.

Prieto Ramos, O. (2008). Envejecimiento y salud. Ciudad de La Habana: Editorial Científico-Técnica.

Teresa A. García Simón. (2005).El envejecimiento: Una nueva etapa de la vida. Facultad de Psicología. UCLV.

Rosa Vega, Y. (Julio de 2011). Propuesta de acciones para la incorporación del adulto mayor a los círculos de abuelos del Consejo Popular "La Posta", municipio Majibacoa. Recuperado el 20 de marzo de 2013, de Contribuciones a las ciencias sociales: www.eumed.net/rev/cccss/13/

Roberto Garcés González. (2012) La gestión del conocimiento en las condiciones del municipio de Remedios como contribución a su desarrollo local. Tesis en opción al Grado Científico de Doctor en Ciencias Sociológicas.

Sánchez, A. (1991). Psicología comunitaria. Barcelona: PPU.

Sandoval Casilimas, C. A. (1996). Investigación Cualitativa. Bogotá: ARFOS.

Valdés Mier, M. A. (1997). Psicogeriatría para Médicos Generales Integrales. Santiago de Cuba: Editorial Oriente. 

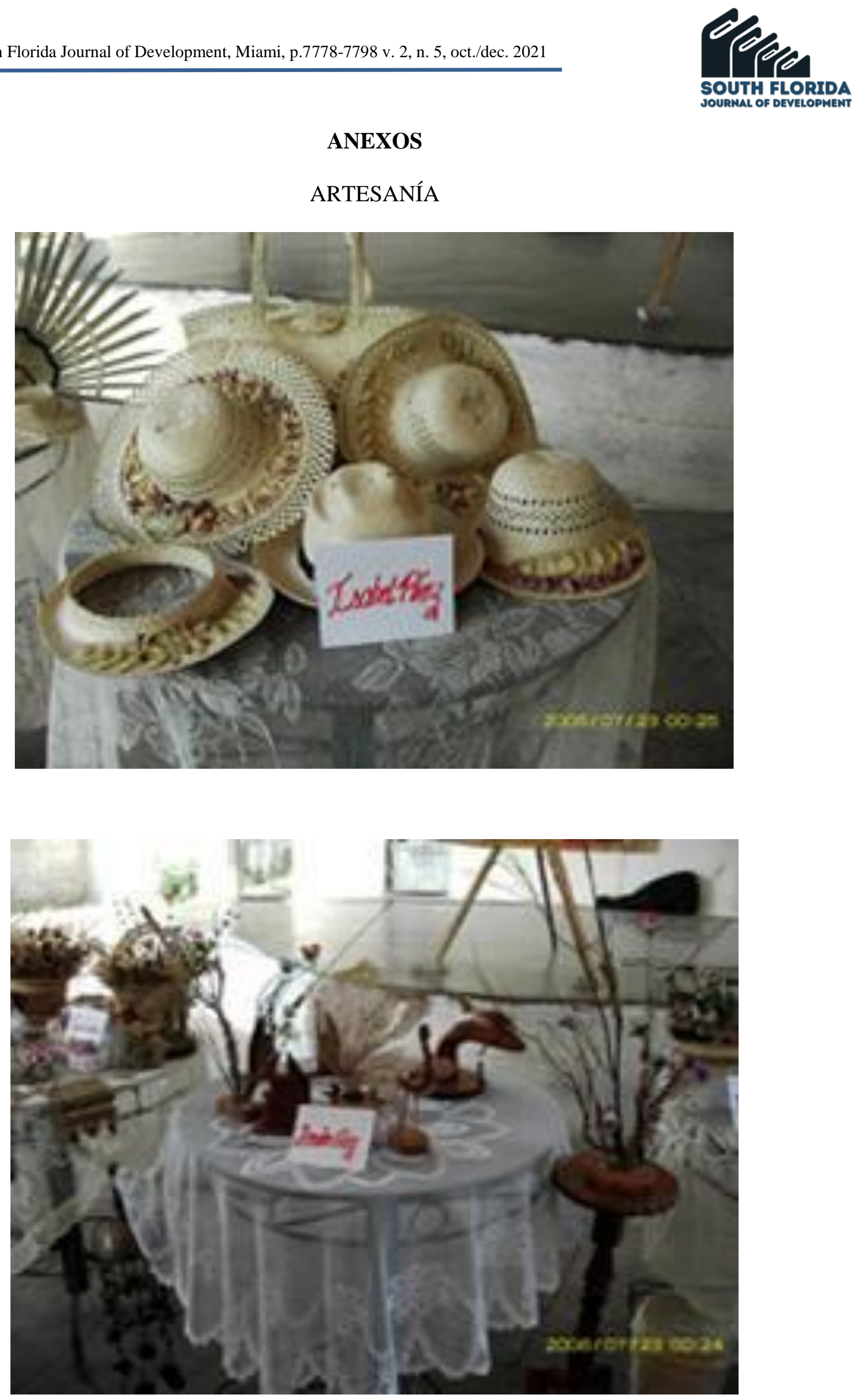

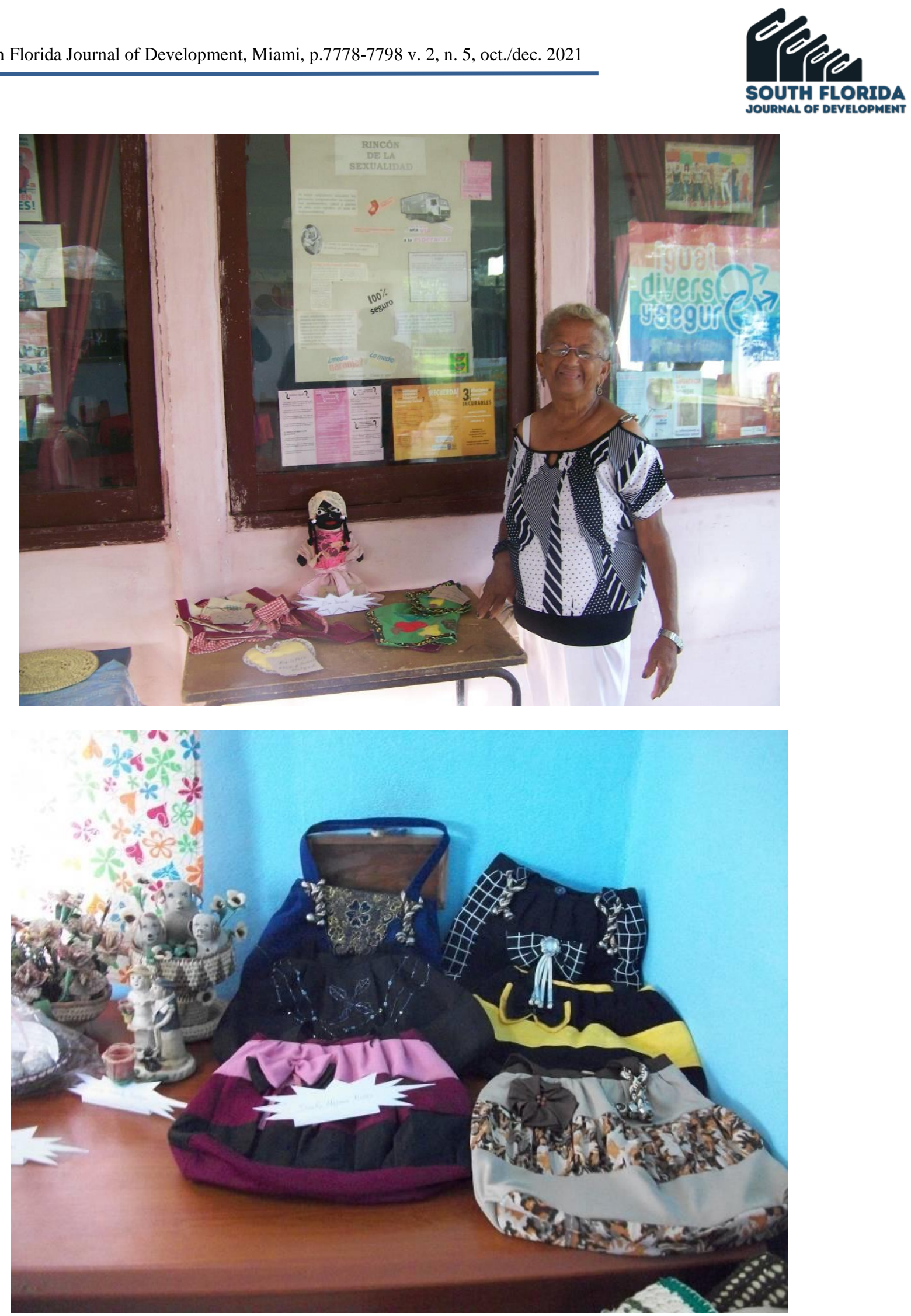

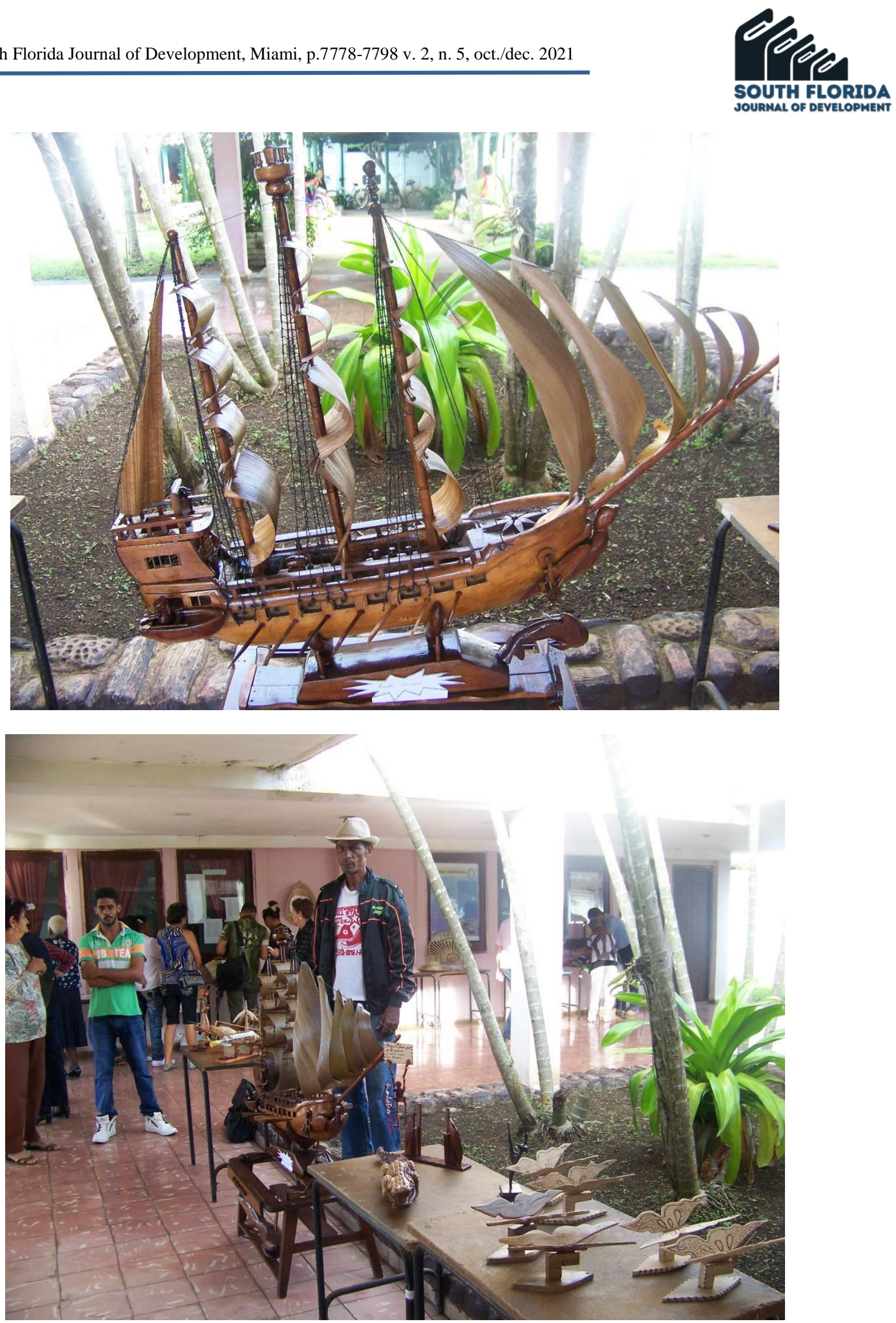


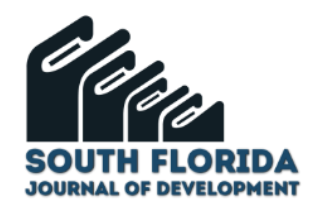

CULTURA
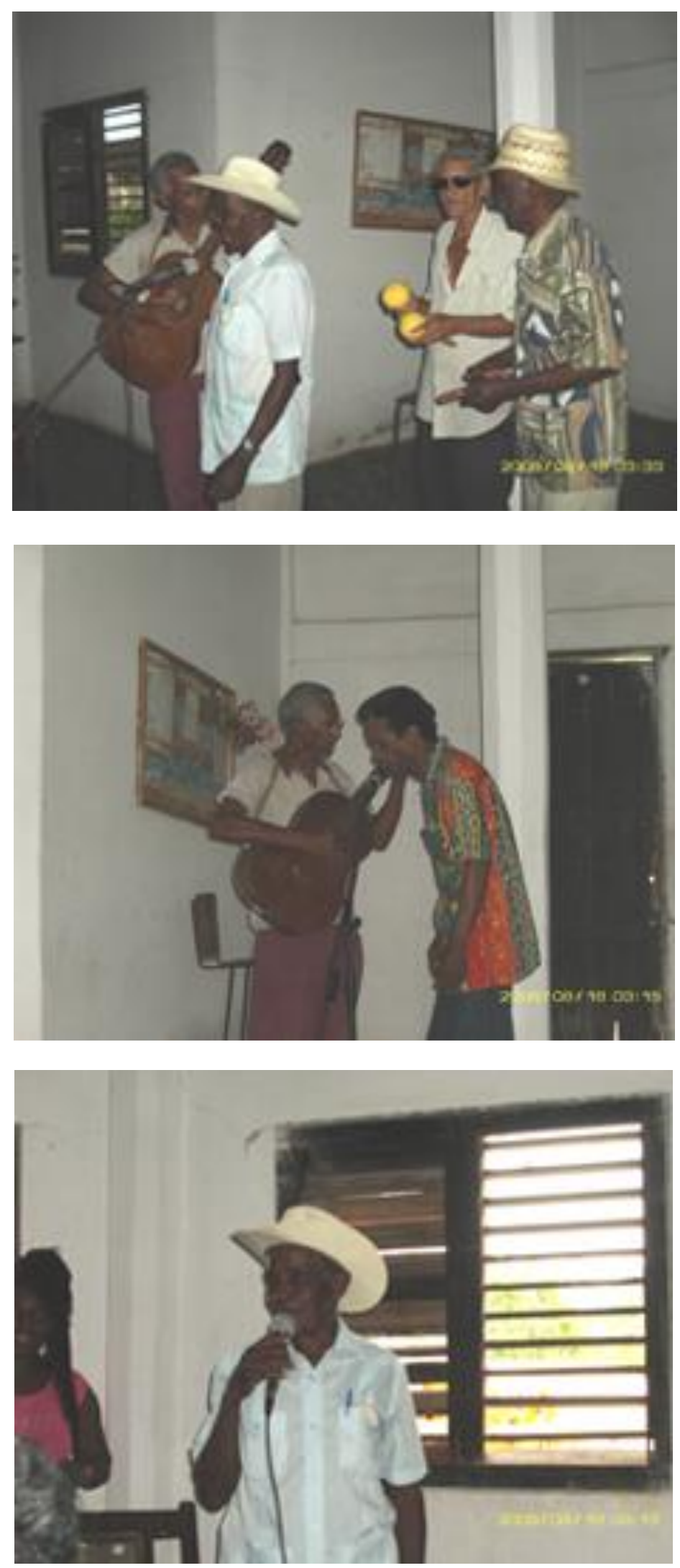


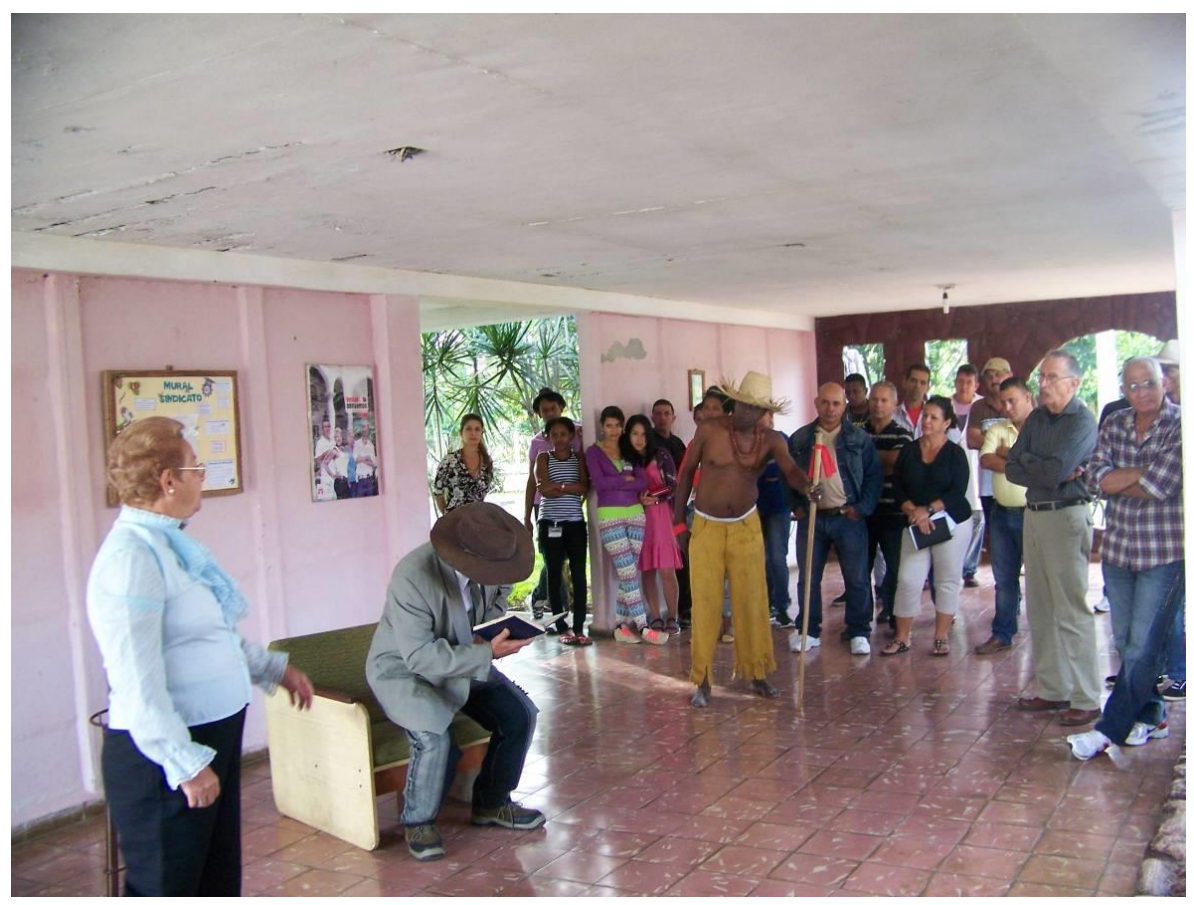

